\title{
Krylov subspace approximations for the exponential Euler method: error estimates and the harmonic Ritz approximant
} E. J. Carr ${ }^{1} \quad$ I. W. Turner ${ }^{2} \quad$ M. Ilic $^{3}$

(Received 28 January 2011; revised 4 August 2011)

\begin{abstract}
We study Krylov subspace methods for approximating the matrixfunction vector product $\varphi(t A) b$ where $\varphi(z)=[\exp (z)-1] / z$. This product arises in the numerical integration of large stiff systems of differential equations by the Exponential Euler Method, where $A$ is the Jacobian matrix of the system. Recently, this method has found application in the simulation of transport phenomena in porous media within mathematical models of wood drying and groundwater flow. We develop an a posteriori upper bound on the Krylov subspace approximation error and provide a new interpretation of a previously published error estimate. This leads to an alternative Krylov approximation to $\varphi(\mathrm{tA}) \mathrm{b}$, the so-called Harmonic Ritz approximant, which we find does not exhibit oscillatory behaviour of the residual error.
\end{abstract}

http://anziamj . austms.org.au/ojs/index.php/ANZIAMJ/article/view/3938 gives this article, (c) Austral. Mathematical Soc. 2011. Published August 8, 2011. ISSN 1446-8735. (Print two pages per sheet of paper.) Copies of this article must not be made otherwise available on the internet; instead link directly to this URL for this article. 


\section{Contents}

1 Introduction

C613

2 Krylov approximation to $\varphi(t A) b$

C615

2.1 An a posteriori error bound . . . . . . . . . . . C615

2.2 New interpretation of error estimate . . . . . . . . . C619

2.3 Harmonic Ritz approximation . . . . . . . . . . . C620

3 Numerical experiments

C623

4 Conclusions

C624

References

C626

\section{Introduction}

Mathematical models for simulating transport phenomena in porous media take the form

$$
\frac{\partial \psi_{\ell}}{\partial t}+\nabla \cdot q_{\ell}=0 \quad \text { on } \Omega,
$$

where $\ell$ denotes the conserved quantity, with appropriate conditions defined on the boundary $\partial \Omega$. For example, a three equation model representing the conservation of water, energy and air is used for modelling the drying of wood [9]. For such problems, the Finite Volume Method (FVM) has been used with great success to solve the governing set of equations. In two dimensions, the domain $\Omega$ is tessellated with triangles and finite volumes are constructed around every node (vertex) in the mesh. For the three equation wood drying model and a mesh comprising of $\mathrm{N}_{\mathrm{p}}$ nodes, the FVM leads to a system of differential equations of the form [2]

$$
\frac{d u}{d t}=g(u), \quad u(0)=u_{0},
$$


where $u \in \mathbb{R}^{3 N_{p}}$ contains the unknown solution values, arranged in triplets, at each node in the mesh. Recently [2], we found that the Exponential Euler Method (EEM) is effective for numerically integrating the resulting differential equation system (1). At each step of the integration process, the EEM solves the linearised system

$$
\frac{d u}{d t}=g\left(u_{n}\right)+J\left(u_{n}\right)\left(u-u_{n}\right), \quad u\left(t_{n}\right)=u_{n},
$$

exactly to obtain the approximate time stepping formula

$$
u_{n+1}=u_{n}+\tau_{n} \varphi\left[\tau_{n} J\left(u_{n}\right)\right] g\left(u_{n}\right),
$$

where $\varphi(z)=[\exp (z)-1] / z, \tau_{n}=t_{n+1}-t_{n}$ is the integration step, and $J$ is the Jacobian matrix of $\mathrm{g}[1,2,7,8]$. This integration strategy is by no means new but it was not until recently [1] that a stepsize control algorithm using local error estimation was provided for the problems of groundwater flow [1] and wood drying [2].

This article focuses on the computation of $\varphi(t A) b$ for the large sparse non-symmetric matrices $A$ encountered in the aforementioned problems; the eigenvalues of these matrices typically have negative real components. Approximations to $\varphi(\mathrm{t} A) \mathrm{b}$ are extracted from the m-dimensional Krylov subspace

$$
\mathcal{K}_{m}(A, b)=\operatorname{span}\left\{b, A b, \ldots, A^{m-1} b\right\} \subseteq \mathbb{R}^{N}, \quad A \in \mathbb{R}^{N \times N},
$$

via Arnoldi's method, which produces the decomposition

$$
A V_{m}=V_{m} H_{m}+\beta_{m} v_{m+1} e_{m}^{\top}, \quad b=\beta_{0} v_{1},
$$

where the columns $v_{1}, v_{2}, \ldots, v_{m}$ of $V_{m} \in \mathbb{R}^{N \times m}$ form an orthonormal basis for $\mathcal{K}_{\mathrm{m}}(A, \mathrm{~b}), \mathrm{H}_{\mathrm{m}}=\mathrm{V}_{\mathrm{m}}^{\top} A V_{\mathrm{m}}\left(\right.$ since $\left.\mathrm{V}_{\mathrm{m}}^{\top} \nu_{\mathrm{m}+1}=0\right), \beta_{0}=\|\mathrm{b}\|_{2}, \beta_{\mathrm{m}}=\|(\mathrm{I}-$ $\left.V_{m} V_{m}^{\top}\right) A v_{m} \|_{2}$ and $e_{m}$ is the $m$ th column of the $m \times m$ identity matrix. The Krylov approximation reduces the evaluation of $\varphi$ to the small $m \times m$ matrix $\mathrm{H}_{\mathrm{m}}[1,2,7,8]$ :

$$
\varphi(t A) b \approx \beta_{0} V_{m} \varphi\left(t H_{m}\right) e_{1}
$$


In practice, beginning at $m=1$, the dimension of the Krylov subspace is increased and the procedure terminated when the approximation (3) is deemed sufficiently accurate. Existing criteria for terminating this approximation procedure are based on the true error [7]:

$$
\varepsilon_{\mathrm{m}}=\varphi(\mathrm{tA}) \mathrm{b}-\beta_{0} \mathrm{~V}_{\mathrm{m}} \varphi\left(\mathrm{tH}_{\mathrm{m}}\right) e_{1} .
$$

Since $\varphi(t A) b$ is unknown, this true error (4) must be estimated or bounded. One error estimate due to Hochbruck, Lubich and Selhofer [7] performs reasonably well in simulation codes $[1,2]$. In Section 2.1 , we attempt to improve upon this error estimate by developing an upper bound on the 2norm of the true error (4) for those matrices encountered in wood drying and groundwater flow applications.

In Section 2.2, we provide a new interpretation of the error estimate of Hochbruck et al. [7] by introducing the concept of a "differential equation residual". This concept relies on the fact that the matrix function $t \varphi(t A) b$ exactly satisfies a suitably defined differential equation. This notion of a residual leads to an alternative Krylov approximation to $\varphi(t A) b$, which we derive by extending methods developed for linear systems that enforce orthogonality of the residual vector and a specified m-dimensional subspace of constraints (see Section 2.3).

\section{Krylov approximation to $\varphi(\mathrm{tA}) \mathrm{b}$}

\subsection{An a posteriori error bound}

Using the Cauchy integral formula, the error of the mth Krylov approximation (4) is represented as [7]

$$
\varepsilon_{\mathrm{m}}=\frac{1}{2 \pi \mathrm{i}} \oint_{\Gamma} \varphi(z)(z \mathrm{I}-\mathrm{t} A)^{-1} r_{\mathrm{m}}(z) \mathrm{d} z
$$




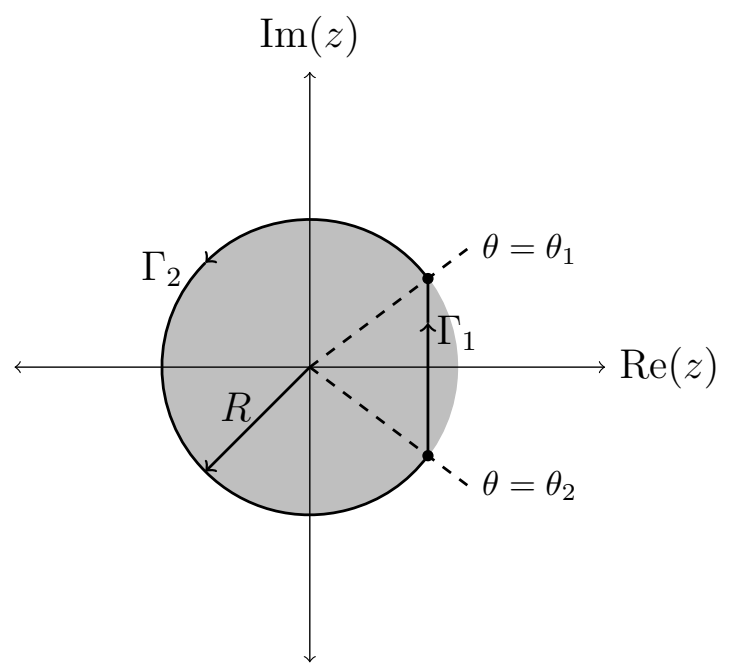

Figure 1: Contour of integration $\Gamma=\Gamma_{1} \cup \Gamma_{2}$ used in the error bound derivation. We take $\Gamma_{1}: z=\alpha+i y, R \sin \left(\theta_{2}\right) \leqslant y \leqslant R \sin \left(\theta_{1}\right)$ and $\Gamma_{2}: z=R e^{i \theta}$, $\theta_{1} \leqslant \theta \leqslant \theta_{2}$ where $\theta_{1}=\arccos (\alpha / R)$ and $\theta_{2}=2 \pi-\arccos (\alpha / R)$.

where the contour of integration $\Gamma$ encloses the eigenvalues of both $t A$ and $t H_{m}$, and where

$$
r_{m}(z)=b-(z I-t A) x_{m}=t \beta_{0} \beta_{m} e_{m}^{\top}\left(z I-t H_{m}\right)^{-1} e_{1} v_{m+1}
$$

is the residual error associated with the Full Orthogonalisation Method (FOM) approximation $x_{m}=\beta_{0} V_{m}\left(z I-t_{m}\right)^{-1} e_{1}$ to the solution of the $z$-shifted linear system $(z \mathrm{I}-\mathrm{t} A) x=\mathrm{b}[7]$.

Our interest concerns matrices $t A$ whose eigenvalues have real components less than some positive value $\alpha$. Consequently, the contour of integration $\Gamma$ is chosen as the boundary of the region formed by the intersection of the disk $|z| \leqslant R$ with the half-plane $\operatorname{Re}(z) \leqslant \alpha$. Taking the limit as $\mathrm{R}$ tends to infinity (to enclose all eigenvalues with real components less than $\alpha$ ), produces the 
following Cauchy integral representation

$$
\varphi(\lambda)=\lim _{R \rightarrow \infty}\left(I_{1}+I_{2}\right), \quad I_{j}=\frac{1}{2 \pi i} \int_{\Gamma_{j}} \frac{\varphi(z)}{z-\lambda} d z, \quad j=1,2,
$$

for $\operatorname{Re}(\lambda)<\alpha$. The second integral in this representation is bounded above:

$$
\begin{aligned}
\left|I_{2}\right| & =\left|\frac{1}{2 \pi} \int_{\theta_{1}}^{\theta_{2}} \frac{\varphi\left(R e^{i \theta}\right)}{R e^{i \theta}-\lambda} R e^{i \theta} d \theta\right| \leqslant \frac{1}{2 \pi} \int_{\theta_{1}}^{\theta_{2}} \frac{e^{R \cos \theta}+1}{R-|\lambda|} d \theta \\
& \leqslant \frac{e^{\alpha}+1}{2 \pi(R-|\lambda|)}\left(\theta_{2}-\theta_{1}\right),
\end{aligned}
$$

where we use $R \cos \theta \leqslant \alpha$ for $\theta_{1} \leqslant \theta \leqslant \theta_{2}$. Taking the limit as $R$ approaches infinity we obtain $\left|\lim _{R \rightarrow \infty} I_{2}\right|=\lim _{R \rightarrow \infty}\left|I_{2}\right|=0$. The significance of this result is that the error representation (5) can be expressed as

$$
\varepsilon_{\mathrm{m}}=\frac{1}{2 \pi} \int_{-\infty}^{\infty} \varphi(\alpha+i \mathrm{y})[(\alpha+i \mathrm{y}) \mathrm{I}-\mathrm{tA}]^{-1} \mathrm{r}_{\mathrm{m}}(\alpha+i \mathrm{y}) \mathrm{d} y .
$$

In the following proposition, we provide an upper bound on the 2-norm of $\varepsilon_{m}$ by considering this integral representation.

Proposition 1. Suppose $\mathrm{A}=\mathrm{PDP}^{-1}$ and $\mathrm{H}_{\mathrm{m}}=\mathrm{Y}_{\mathrm{m}} \Lambda_{\mathrm{m}} \mathrm{Y}_{\mathrm{m}}^{-1}$ are diagonalisable with eigenvalues $\lambda_{j}, j=1, \ldots, N$, and $\mu_{k}, k=1, \ldots, m$. Furthermore, take $\lambda_{\max }$ to be the maximum real component of the eigenvalues of $\mathrm{A}$. Then for $\alpha$ positive and $\alpha>\mathrm{t} \lambda_{\max }$, the 2-norm of the Krylov approximation error $\varepsilon_{\mathrm{m}}=\varphi(\mathrm{tA}) \mathrm{b}-\beta_{0} \mathrm{~V}_{\mathrm{m}} \varphi\left(\mathrm{tH}_{\mathrm{m}}\right) \mathrm{e}_{1}$ satisfies

$$
\left\|\varepsilon_{m}\right\|_{2} \leqslant \mathcal{C}_{m}(\alpha)\left\|r_{m}(\alpha)\right\|_{2}
$$

where

$$
\mathcal{C}_{m}(\alpha)=\frac{\kappa_{2}(P)\left(e^{\alpha}+1\right)}{2 \alpha^{1 / 2}\left(\alpha-t \lambda_{\max }\right)^{1 / 2}} \prod_{k=1}^{m}\left[1+\left(\frac{\mathrm{t} \operatorname{Im}\left(\mu_{k}\right)}{\alpha-\mathrm{t} \operatorname{Re}\left(\mu_{k}\right)}\right)^{2}\right]^{1 / 2},
$$

$\mathrm{K}_{2}(\mathrm{P})$ is the 2-norm condition number of $\mathrm{P}$, and $\mathrm{r}_{\mathrm{m}}(\alpha)$ is the residual error associated with the FOM approximation to $(\alpha \mathrm{I}-\mathrm{tA})^{-1} \mathrm{~b}$. 
Proof: Taking norms of (7) gives

$$
\left\|\varepsilon_{m}\right\|_{2} \leqslant \frac{1}{2 \pi} \int_{-\infty}^{\infty}|\varphi(\alpha+i y)|\left\|((\alpha+i y) I-t A)^{-1}\right\|_{2}\left\|r_{m}(\alpha+i y)\right\|_{2} d y .
$$

We make use of the results

$$
\begin{gathered}
|\varphi(\alpha+i y)| \leqslant \frac{e^{\alpha}+1}{|\alpha+i y|}, \\
\left\|((\alpha+i y) I-t A)^{-1}\right\|_{2} \leqslant \kappa_{2}(P) \max _{j=1, \ldots, N} \frac{1}{\left|(\alpha+i y)-t \lambda_{j}\right|},
\end{gathered}
$$

and

$$
\begin{aligned}
\left\|r_{m}(\alpha+i y)\right\|_{2} & =\prod_{k=1}^{m} \frac{\left|\alpha-t \mu_{k}\right|}{\left|\alpha+i y-t \mu_{k}\right|}\left\|r_{m}(\alpha)\right\|_{2}, \\
& \leqslant \prod_{k=1}^{m}\left[1+\left(\frac{t \operatorname{Im}\left(\mu_{k}\right)}{\alpha-t \operatorname{Re}\left(\mu_{k}\right)}\right)^{2}\right]^{1 / 2}\left\|r_{m}(\alpha)\right\|_{2} .
\end{aligned}
$$

The third result follows from expressing $e_{m}^{\top}\left(z \mathrm{I}-\mathrm{tH}_{\mathrm{m}}\right)^{-1} e_{1}$ in the definition of the residual vector (6) in adjoint-determinant form for both $z=\alpha+i y$ and $z=\alpha$ and then taking norms. Using these results one obtains

$$
\begin{aligned}
\left\|\varepsilon_{m}\right\|_{2} \leqslant & \kappa_{2}(P)\left(e^{\alpha}+1\right) \prod_{k=1}^{m}\left[1+\left(\frac{t \operatorname{Im}\left(\mu_{k}\right)}{\alpha-t \operatorname{Re}\left(\mu_{k}\right)}\right)^{2}\right]^{1 / 2} \\
& \quad \times \max _{j=1, \ldots, N} \int_{-\infty}^{\infty} \frac{1}{|\alpha+i y|} \frac{1}{\left|(\alpha+i y)-t \lambda_{j}\right|} d y\left\|r_{m}(\alpha)\right\|_{2} .
\end{aligned}
$$

The integral is then bounded above using the Cauchy-Schwarz Inequality:

$$
\begin{aligned}
I & =\max _{j=1, \ldots, N} \int_{-\infty}^{\infty} \frac{1}{|\alpha+i y|} \frac{1}{\left|(\alpha+i y)-t \lambda_{j}\right|} d y \\
& \leqslant \max _{j=1, \ldots, N}\left(\int_{-\infty}^{\infty} \frac{1}{|\alpha+i y|^{2}} d y\right)^{1 / 2}\left(\int_{-\infty}^{\infty} \frac{1}{\left|\alpha+i y-t \lambda_{j}\right|^{2}} d y\right)^{1 / 2}
\end{aligned}
$$




$$
=\max _{j=1, \ldots, N}\left(\frac{\pi}{\alpha}\right)^{1 / 2}\left(\frac{\pi}{\alpha-\mathrm{t} \operatorname{Re}\left(\lambda_{j}\right)}\right)^{1 / 2}
$$

Remark 2. In the case when the matrix is not diagonalisable, one must use the Jordan canonical form [5]. This will be the subject of future research.

Proposition 1 provides an upper bound that can be used to terminate the approximation procedure. However, it requires knowledge of the values of $\kappa_{2}(\mathrm{P})$ and $\lambda_{\max }$. A practical error estimate based on this bound is obtained by estimating these values using the projected matrix $\mathrm{H}_{\mathrm{m}}$, via the approximations $\kappa_{2}(\mathrm{P}) \approx \kappa_{2}\left(\mathrm{Y}_{\mathrm{m}}\right)$ and $\lambda_{\max } \approx \mu_{\max }=\max _{\mathrm{k}=1, \ldots, \mathrm{m}} \operatorname{Re}\left(\mu_{\mathrm{k}}\right)$. This produces the estimate $\mathcal{C}_{\mathrm{m}}(\alpha) \approx \tilde{\mathcal{C}}_{\mathrm{m}}(\alpha)$ for use in (8) where

$$
\tilde{\mathcal{C}}_{\mathrm{m}}(\alpha)=\frac{\kappa_{2}\left(Y_{m}\right)\left(e^{\alpha}+1\right)}{2 \alpha^{1 / 2}\left(\alpha-t \mu_{\max }\right)^{1 / 2}} \prod_{k=1}^{m}\left[1+\left(\frac{t \operatorname{Im}\left(\mu_{k}\right)}{\alpha-t \operatorname{Re}\left(\mu_{k}\right)}\right)^{2}\right]^{1 / 2} \text {. }
$$

\subsection{New interpretation of error estimate}

Firstly, we briefly outline the estimate of the true error (4) given by Hochbruck et al. [7]. Consider the Cauchy integral representation (5), expressed as

$$
\varepsilon_{\mathrm{m}}=\frac{1}{2 \pi \mathrm{i}} \int_{\Gamma} \varphi(z) \epsilon_{\mathrm{m}}(z) \mathrm{d} z
$$

where $\epsilon_{\mathrm{m}}(z)=x-x_{\mathrm{m}}$ is the true error associated with the FOM approximation $x_{m}=\beta_{0} V_{m}\left(z I-t H_{m}\right)^{-1} e_{1}$ to the solution of the $z$-shifted linear system $(z \mathrm{I}-\mathrm{tA}) \mathrm{x}=\mathrm{b}$. Hochbruck et al. [7] argued that since the termination of FOM for this linear system is typically based on the residual error $r_{m}(z)$ rather than the true error $\epsilon_{\mathfrak{m}}(z)$, one substitutes for $\epsilon_{\mathfrak{m}}(z)$ the error indicator $r_{m}(z)$ (6) giving

$$
\varepsilon_{\mathrm{m}} \approx \frac{1}{2 \pi \mathrm{i}} \int_{\Gamma} \varphi(z) \mathrm{r}_{\mathrm{m}}(z) \mathrm{d} z=\mathrm{t} \beta_{0} \beta_{\mathrm{m}} e_{\mathrm{m}}^{\top} \varphi\left(\mathrm{t} \mathrm{H}_{\mathrm{m}}\right) e_{1} v_{\mathrm{m}+1}
$$


We now provide a new interpretation of the resulting error estimate by introducing the concept of a differential equation residual. This relies on the fact that the function $x(t)=t \varphi(t A) b=A^{-1}\left(e^{t A}-I\right) b$ satisfies

$$
\frac{d x}{d t}=A x+b, \quad x(0)=0 .
$$

We replace $\varphi(t A) b$ by its Krylov approximation (3) and hence define the approximation $x_{m}(t)=t \beta_{0} V_{m} \varphi\left(t H_{m}\right) e_{1}=\beta_{0} V_{m} H_{m}^{-1}\left(e^{t H_{m}}-I\right) e_{1}$. One way to measure how well $x_{m}$ approximates $x$ (and hence determine the accuracy of the Krylov approximation (3)) is to measure how well $x_{m}$ satisfies the differential equation (11). We measure this through the "differential equation residual", defined as

$$
\rho_{\mathrm{m}}=\mathrm{b}+A x_{\mathrm{m}}-\frac{d x_{\mathrm{m}}}{\mathrm{dt}}
$$

where $\rho_{m}=0$ when $x_{m}=x$ and one assumes a small value of $\left\|\rho_{m}\right\|$ means $x_{m}$ is a good approximation to $x$. A similar error interpretation was given for the matrix exponential by Celledoni and Moret [4]. Making use of the Arnoldi decomposition (2) and noting that $b=\beta_{0} V_{m} e_{1}$, one obtains

$$
\begin{aligned}
& \rho_{\mathrm{m}}=\mathrm{b}+\mathrm{t} \beta_{0} A V_{\mathrm{m}} \varphi\left(\mathrm{tH} \mathrm{H}_{\mathrm{m}}\right) e_{1}-\beta_{0} \mathrm{~V}_{\mathrm{m}} e^{\mathrm{t} \mathrm{H}_{\mathrm{m}}} e_{1} \\
& =\mathrm{b}+\mathrm{t} \beta_{0} A V_{\mathrm{m}} \varphi\left(\mathrm{t} \mathrm{H}_{\mathrm{m}}\right) e_{1}-\beta_{0} \mathrm{~V}_{\mathrm{m}}\left(\mathrm{tH}_{\mathrm{m}} \varphi\left(\mathrm{tH}_{\mathrm{m}}\right)+\mathrm{I}_{\mathrm{m}}\right) e_{1} \\
& =\mathrm{t} \beta_{0}\left(A V_{\mathrm{m}}-\mathrm{V}_{\mathrm{m}} \mathrm{H}_{\mathrm{m}}\right) \varphi\left(\mathrm{tH}_{\mathrm{m}}\right) e_{1} \\
& =t \beta_{0} \beta_{m} e_{m}^{T} \varphi\left(t H_{m}\right) e_{1} v_{m+1},
\end{aligned}
$$

as a measure of the accuracy of the approximation (3), which is identical to the error estimate proposed by Hochbruck et al. [7]. As we see in the next section, this error interpretation can be used to construct an alternative Krylov approximant to $\varphi(\mathrm{tA}) \mathrm{b}$.

\subsection{Harmonic Ritz approximation}

For linear systems, Krylov projection methods extract an approximate solution from $\mathcal{K}_{\mathrm{m}}$ by forcing the residual vector to be orthogonal to an $\mathrm{m}$-dimensional 
subspace of constraints $\mathcal{W}_{\mathrm{m}} \subseteq \mathbb{R}^{\mathrm{N}}$. We extend this idea to the "differential equation residual" introduced in Section 2.2 to produce an alternative Krylov approximation to $\varphi(t A) b$.

First, each vector $x_{m} \in \mathcal{K}_{m}$ is expressible in the form $x_{m}=V_{m} y_{m}$ where $y_{m} \in \mathbb{R}^{m}$. This produces the general form of the residual vector (12)

$$
\rho_{m}=b+A V_{m} y_{m}-V_{m} \frac{d y_{m}}{d t},
$$

where we assume $y_{m}$ is a function of $t$.

To produce the FOM approximate solution of a linear system, one chooses $\mathcal{W}_{\mathrm{m}}=\mathcal{K}_{\mathrm{m}}$. Interestingly, forcing $\rho_{\mathrm{m}}$ to be orthogonal to $\mathcal{K}_{\mathrm{m}}$, that is $\bigvee_{\mathrm{m}}^{\top} \rho_{\mathrm{m}}=$ 0 , we obtain

$$
\begin{array}{r}
V_{m}^{\top} b+\left(V_{m}^{\top} A V_{m}\right) y_{m}-\left(V_{m}^{\top} V_{m}\right) \frac{d y_{m}}{d t}=0 \\
\beta_{0} e_{1}+H_{m} y_{m}-\frac{d y_{m}}{d t}=0
\end{array}
$$

due to the columns of $V_{m}$ forming an orthonormal basis. The solution of this differential equation is $y_{m}=t \beta_{0} \varphi\left(t H_{m}\right) e_{1}$ and hence $x_{m}=t \beta_{0} V_{m} \varphi\left(t H_{m}\right) e_{1}$, which reproduces the Krylov approximation defined by (3).

Another Krylov projection method for linear systems, the Generalised Minimal Residual Method (GMRES), is often preferred over FOM since the resulting approximate solution minimises the 2 -norm of the residual vector over $\mathcal{K}_{m}$. For linear systems, this choice of the constraint space $\mathcal{W}_{m}$ is well known; however, the choice that minimises the 2 -norm of $\rho_{m}$ is not as straightforward. As a result, we take $\mathcal{W}_{\mathrm{m}}=A \mathcal{K}_{\mathrm{m}}$, which produces the GMRES approximate solution to the linear system $A x=b$. Forcing $\rho_{m}$ to be orthogonal to $A \mathcal{K}_{m}$ requires

$$
\left(A V_{m}\right)^{\top}\left(b+A V_{m} y_{m}-V_{m} \frac{d y_{m}}{d t}\right)=0
$$


Using the Arnoldi decomposition (2),

$\left(V_{m} H_{m}+\beta_{m} v_{m+1} e_{m}^{T}\right)^{T}\left[\beta_{0} V_{m} e_{1}+\left(V_{m} H_{m}+\beta_{m} v_{m+1} e_{m}^{T}\right) y_{m}-V_{m} \frac{d y_{m}}{d t}\right]=0$ and given that $v_{m+1}$ is orthogonal to each column of $V_{m}$, we obtain

$$
\beta_{0} H_{m}^{T} e_{1}+\left(H_{m}^{T} H_{m}+\beta_{m}^{2} e_{m} e_{m}^{T}\right) y_{m}-H_{m}^{T} \frac{d y_{m}}{d t}=0 .
$$

Assuming $H_{m}^{\top}$ is invertible and denoting $H_{m}^{-\top}=\left(H_{m}^{\top}\right)^{-1}$ one obtains

$$
\beta_{0} e_{1}+\left(H_{m}+\beta_{m}^{2} H_{m}^{-T} e_{m} e_{m}^{T}\right) y_{m}-\frac{d y_{m}}{d t}=0
$$

The solution of this differential equation is $y_{m}=t \beta_{0} \varphi\left(t \mathcal{H}_{m}\right) e_{1}$ where $\mathcal{H}_{m}=$ $\mathrm{H}_{\mathrm{m}}+\beta_{\mathrm{m}}^{2} \mathrm{H}_{\mathrm{m}}^{-\mathrm{T}} e_{\mathrm{m}} e_{\mathrm{m}}^{\top}$ and hence $x_{\mathrm{m}}=\mathrm{t} \beta_{0} \mathrm{~V}_{\mathrm{m}} \varphi\left(\mathrm{t} \mathcal{H}_{\mathrm{m}}\right) e_{1}$. This defines the alternative Krylov approximation

$$
\varphi(t A) b \approx \beta_{0} V_{m} \varphi\left(t \mathcal{H}_{m}\right) e_{1}, \quad \mathcal{H}_{m}=H_{m}+\beta_{m}^{2} f_{m} e_{m}^{T},
$$

where the evaluation of $\varphi$ occurs at a matrix given by a rank one update on $\mathrm{H}_{m}$ and $f_{m}=H_{m}^{-\top} e_{m}$. We refer to the case $f_{m}=H_{m}^{-\top} e_{m}$ as the harmonic Ritz approximant since the eigenvalues of $\mathcal{H}_{\mathfrak{m}}$ are the harmonic Ritz values of $\mathrm{A}$ with respect to the subspace $\mathcal{K}_{m}$. A generalised version of (14) was derived by Hochbruck and Hochstenbach [6] using a different strategy. For (14), the "differential equation residual" is defined as

$$
\begin{aligned}
\rho_{\mathrm{m}} & =\mathrm{b}+\mathrm{t} \beta_{0} A V_{\mathrm{m}} \varphi\left(\mathrm{t} \mathcal{H}_{\mathrm{m}}\right) e_{1}-\beta_{0} V_{\mathrm{m}} e^{\mathrm{t} \mathcal{H}_{\mathrm{m}}} e_{1}, \\
& =\mathrm{b}+\mathrm{t} \beta_{0} A V_{\mathrm{m}} \varphi\left(\mathrm{t} \mathcal{H}_{\mathrm{m}}\right) e_{1}-\beta_{0} V_{\mathrm{m}}\left(\mathrm{t} \mathcal{H}_{\mathrm{m}} \varphi\left(\mathrm{t} \mathcal{H}_{\mathrm{m}}\right)+\mathrm{I}_{\mathrm{m}}\right) e_{1} \\
& =\mathrm{t} \beta_{0}\left(A V_{\mathrm{m}}-V_{\mathrm{m}} \mathcal{H}_{\mathrm{m}}\right) \varphi\left(\mathrm{t} \mathcal{H}_{\mathrm{m}}\right) e_{1}, \\
& =\mathrm{t} \beta_{0} \beta_{\mathrm{m}} e_{\mathrm{m}}^{T} \varphi\left(\mathrm{t} \mathcal{H}_{\mathrm{m}}\right) e_{1}\left[v_{\mathrm{m}+1}-\beta_{\mathrm{m}} \mathrm{V}_{\mathrm{m}} \mathrm{f}_{\mathrm{m}}\right] .
\end{aligned}
$$

Setting $f_{m}=0$ in Equations (14) and (15) produces the standard Krylov approximation, which we refer to in the next section as the Ritz approximant. 


\section{Numerical experiments}

All numerical experiments were conducted in MATLAB Version 7.1 based on a single representative matrix $A=J\left(u_{n}\right)$ of size $1899 \times 1899$ and vector $\mathrm{b}=\mathrm{g}\left(\mathrm{u}_{\mathrm{n}}\right)$ extracted from a low temperature, wood drying, simulation $[2,3]$. The real and imaginary eigenvalue components of $A$ range from $-10^{2}$ to $-7.4 \times 10^{-5}$ and $-5.0 \times 10^{-4}$ to $5.0 \times 10^{-4}$, respectively.

First, we assess the performance of the error bound given in (8) and (9) of Section 2.1 and the error estimate defined by (10) for a time step of $t=200$ (Figure 2). Recall that the error estimate is obtained by using the approximations $\kappa_{2}(\mathrm{P}) \approx \mathrm{\kappa}_{2}\left(\mathrm{Y}_{\mathrm{m}}\right)$ and $\lambda_{\max }=\mu_{\max } \approx \max _{k=1, \ldots, \mathrm{m}} \operatorname{Re}\left(\mu_{\mathrm{k}}\right)$ in the bound. In these results, $\alpha>0$ can be freely chosen. We find increasing the value of $\alpha$ provides a sharper bound for large $m$ but a poorer bound for small $\mathrm{m}$. This trade-off occurs due to two reasons: the appearance of the term $\exp (\alpha)$ in the constants $\mathcal{C}_{\mathfrak{m}}(\alpha)$ and $\tilde{\mathcal{C}}_{m}(\alpha)$; and the faster convergence of the linear system residual $r_{m}(\alpha)$ for larger $\alpha$. However, for both the error bound and error estimate, a value of $\alpha$ could not be found that improved upon the "differential equation residual" error (estimate of Hochbruck et al. [7]).

We now assess the performance of both the Ritz and harmonic Ritz approximants to $\varphi(t A) b$, defined in (14) with $f_{m}=0$ and $f_{m}=H_{m}^{-\top} e_{m}$ respectively (Figure 3). Eigenvalue decompositions are used to compute both $\varphi\left(\mathrm{tH}_{\mathrm{m}}\right)$ and $\varphi\left(\mathrm{tH}_{\mathrm{m}}\right)$. Our initial experiments discovered that for some values of $\mathrm{m}$, a single positive harmonic Ritz value was produced that eroded the accuracy of the given harmonic Ritz approximant. We found it necessary to discard these eigenvalues by approximating $\varphi\left(t \mathcal{H}_{\mathrm{m}}\right)$ by

$$
\varphi\left(\mathrm{t} \mathcal{H}_{\mathrm{m}}\right) \approx \sum_{\operatorname{Re}\left(\theta_{\mathrm{j}}\right)<0} \varphi\left(\mathrm{t} \theta_{j}\right) y_{j} z_{j}^{\top}, \quad \mathcal{H}_{\mathrm{m}} \mathrm{Y}_{\mathrm{m}}=\Theta_{\mathrm{m}} \mathrm{Y}_{\mathrm{m}}
$$

where $y_{j}$ is the $j$ th column of $Y_{m}$ such that $\left(\theta_{j}, y_{j}\right)$ is an eigenpair of $\mathcal{H}_{m}$ and $z_{j}^{\top}$ is the $j$ th row of $Y_{m}^{-1}$. Time steps of $t=50$ and $t=200$ (slower convergence) 


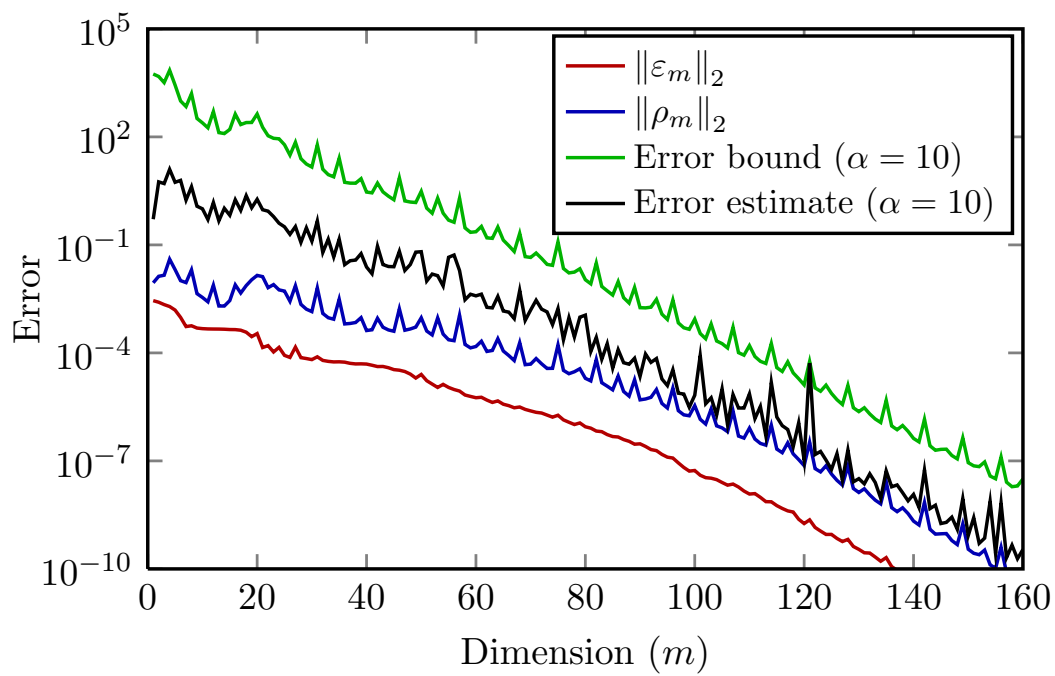

Figure 2: Accuracy of error estimates and error bounds to $\varepsilon_{\mathrm{m}}=\varphi(\mathrm{t} A) \mathrm{b}-$ $\beta_{0} V_{m} \varphi\left(t H_{m}\right) e_{1}$ for a time step of $t=200$. Comparison of the "differential equation residual" (13), error bound (given in Equations (8) and (9)) and error estimate defined by (10).

were both tested. From the plot, the behaviour of the "differential equation residual" error for the harmonic Ritz approximant is more favourable than the standard Ritz approximant. The former eliminates the oscillation, providing a smooth monotone decreasing residual error.

\section{Conclusions}

We derived an a posteriori upper bound on the Krylov subspace approximation error for the matrix-function vector product $\varphi(t A) b$. This error bound provides a mechanism through which the quality of the Krylov approximant can be assessed as the dimension of the subspace is increased. We also 


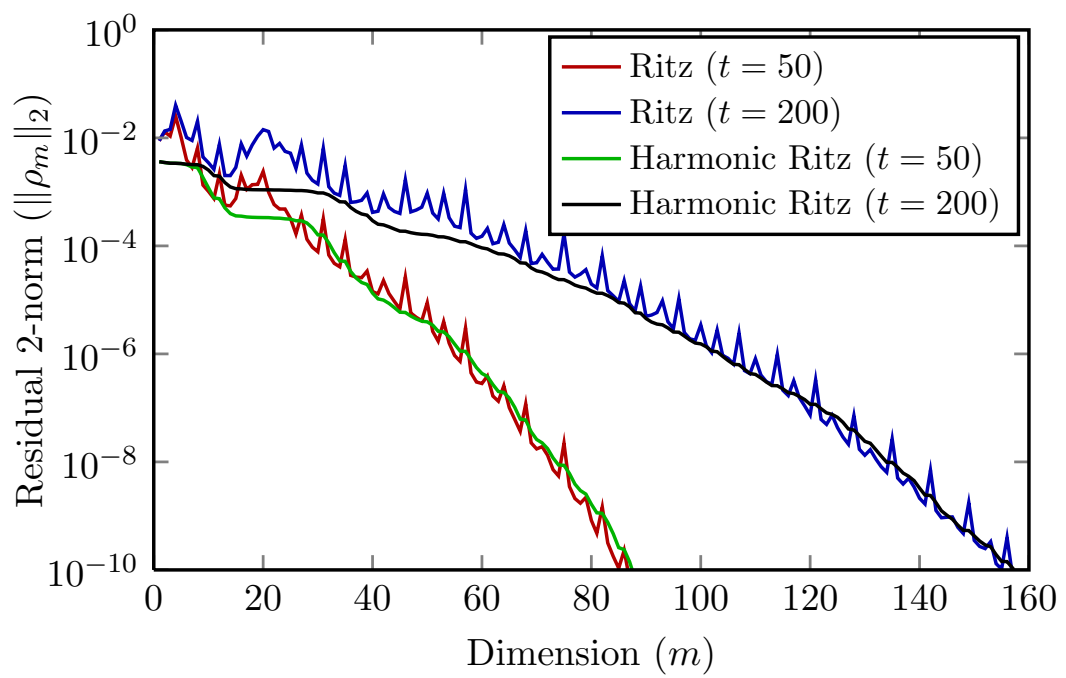

Figure 3: Accuracy of Krylov approximants to $\varphi(t A) b$. Comparison of the "differential equation residual" error (15) for the Ritz $\left(f_{m}=0\right)$ and Harmonic $\operatorname{Ritz}\left(f_{m}=H_{m}^{-T} e_{m}\right)$ approximants for two values of the time step $t$.

introduced the concept of a "differential equation residual" and used this definition to explain why a previously defined error estimate performs adequately in predicting when to terminate the Krylov subspace approximation procedure. This finding identifies an alternative Krylov subspace approximation to $\varphi(t A) b$ that provides "smoother" residual errors than the standard approximation. We believe this alternative approximation has potential and our intention is to utilise this result in future versions of our Exponential Euler Method (EEM) simulation code for further case studies in modelling transport processes in porous media. 


\section{References}

[1] E. J. Carr, T. J. Moroney and I. W. Turner. Efficient simulation of unsaturated flow using exponential time integration. Appl. Math. Comput., 217(14):6587-6596, 2011. doi:10.1016/j.amc.2011.01.041 C614, C615

[2] E. J. Carr, I. W. Turner and P. Perré. A Jacobian-free exponential integrator for simulating transport in heterogeneous porous media: application to the drying of softwood, submitted for publication. C613, C614, C615, C623

[3] E. J. Carr, I. W. Turner and P. Perré. A new control-volume finite-element scheme for heterogeneous porous media:application to the drying of softwood. Chem. Eng. Technol., 34(7):1143-1150, 2011. doi:10.1002/ceat.201100060 C623

[4] E. Celledoni and I. Moret. A Krylov projection method for systems of ODEs. Appl. Numer. Math., 24(2-3):365-378, 1997. doi:10.1016/S0168-9274(97)00033-0 C620

[5] N. J. Higham. Functions of matrices: theory and computation. SIAM, Philadelphia, PA, USA, 2008. C619

[6] M. Hochbruck, M. E. Hochstenbach. Subspace extraction for matrix functions, submitted for publication. http://www.win.tue.nl/ hochsten/pdf/funext.pdf C622

[7] M. Hochbruck, C. Lubich and H. Selhofer. Exponential integrators for large systems of differential equations. SIAM J. Sci. Comput., 19(5):1552-1574, 1998. doi:10.1137/S1064827595295337 C614, C615, C616, C619, C620, C623

[8] B. V. Minchev and W. M. Wright. A review of exponential integrators for first order semi-linear problems. Numerics No. 2/05, Norwegian University of Science and Technology, Trondheim, Norway, 2005. 
http: //www . math.ntnu.no/preprint/numerics/2005/N2-2005.ps C614

[9] P. Perré and I. Turner. A heterogeneous wood drying computational model that accounts for material property variation across growth rings. Chem. Eng. J., 86(1-2):117-131, 2002. doi:10.1016/S1385-8947(01)00270-4 C613

\section{Author addresses}

1. E. J. Carr, Mathematical Sciences, Queensland University of Technology, Brisbane, Australia. mailto:elliot.carr@qut.edu.au

2. I. W. Turner, Mathematical Sciences, Queensland University of Technology, Brisbane, Australia. mailto:i.turner@qut.edu.au

3. M. Ilic, Mathematical Sciences, Queensland University of Technology, Brisbane, Australia. mailto:m.ilic@qut.edu.au 